\title{
Aplicación de un instrumento para medir el grado de liderazgo en el hotel Villa Cuba
}

DOI: https://doi.org/10.33262/ap.v1i1.18

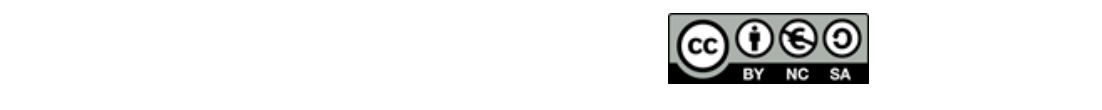

The use of an instrument to measure the leadership degree on the Villa Cuba Hotel

Darien Mesa Ramos., ${ }^{1}$ Bisleivys Jiménez Valero., ${ }^{2}$ \& Yunislay Bordón Gómez. ${ }^{3}$

\begin{abstract}
.
Tourism is the main driving economic force of other economic activities and it grows as time passes, however at present, there is not enough knowledge and necessary preparation regarding Technology Management and innovation in touristic services.

This work was carried with the main objective of measuring the leadership degree of said institution, to do so the appropriate instrument was applied; an instrument considered innovative on the scientific field. It consists on the deployment of a questionnaire which was developed by DETUM professors of the UMCC.

The use of this methodology proved the perceived leadership value to be low, the workers are not motivated and a good leadership strategy is not in place, resulting in a poor performance, because of this comes the necessity to evaluate and propose measures and objectives to further improve their work.
\end{abstract}

Keywords: instrument, leadership, management, technology, innovation.

\footnotetext{
${ }^{1}$ Universidad de Matanzas. Cuba. bisleivys.jimenez@umcc.cu

${ }^{2}$ Universidad de Matanzas. Cuba. bisleivys.jimenez@umcc.cu

${ }^{3}$ Universidad de Matanzas. Cuba. yunislaybg@gmail.com
} 


\section{Resumen.}

El turismo es la actividad impulsora de otras actividades económicas y sigue creciendo, sin embargo, en la actualidad no existe el conocimiento y la preparación necesaria en cuanto a la Gestión de la Tecnología y la innovación en los servicios turísticos.

Este trabajo se realizó con el objetivo de medir el grado de liderazgo en la entidad objeto de estudio, para ello se utilizó un instrumento que es una novedad científica y radica en la puesta en práctica de un cuestionario hecho por los profesores del CETUM de la UMCC.

El uso de esta metodología evidenció que el valor percibido del liderazgo es bajo, los trabajadores no son motivados y no se ejerce un buen liderazgo, por lo que no tienen un buen rendimiento, es por ello que es necesario evaluar y proponer medidas y objetivos para mejorar su accionar.

Palabras claves: herramienta, liderazgo, gestión, tecnología, innovación.

\section{Introducción.}

El desarrollo de la actividad turística se ha convertido en muchos países en una de sus principales ramas de la economía, hoy en día son pocos los países que no explotan, ya sea, sus atractivos naturales, arquitectónicos o culturales, ya que el turismo es un factor realmente importante para el desarrollo socioeconómico y cultural de un país, dada la diversidad de actividades favorables que traen bonanzas económicas: es un instrumento generador de divisas, al ser una actividad que canaliza una inversión para producir una expansión económica general; genera asimismo un mercado de empleos diversificado con una inversión relativamente baja en comparación con otros sectores de la economía; genera una balanza de pagos favorables y sobre todo desarrolla las actividades económicas locales.

El Turismo es un medio de intercambio social, ya que muestra efectivamente que la sociedad que desarrolla más la actividad del turismo se capacita hacia la comprensión, para la adaptación de sus medios habituales, juzgando de una manera positiva su propia sociedad, estos factores si se suman al desarrollo tecnológico del ferrocarril, el automóvil, el avión y de la navegación, ayudan a que haya dejado de ser una opción de grupos selectivos y que en la actualidad un gran número de personas participen del mismo.

El mundo en que esta actividad se desarrolla es muy volátil, ya que son muchos los competidores y la competencia crece cada vez más. El éxito de la industria turística está basado en la generación y la transmisión de conocimientos, una de las formas de diferenciarse de los competidores es innovando, la innovación consiste en la conversión del conocimiento tecnológico en nuevos productos, nuevos servicios o procesos para su introducción en el mercado, así como los cambios tecnológicamente significativos en los productos, servicios y procesos. Para que exista innovación no basta solo con que se 
investigue, sino que el resultado de esta investigación debe introducirse en el mercado, de manera que sea comercializable y factible para la entidad. Esto pasa en la actividad turística cuando se logra poner en práctica una serie de factores combinados como la investigación, la capacidad de los trabajadores del sector para asimilar y comunicar dichos conocimientos y la capacidad de la empresa para tomar estas nuevas tecnologías y conocimientos.

Las empresas turísticas para sobrevivir deben realizar una buena gestión de la tecnología y la innovación, midiendo la capacidad innovadora a través del grado de liderazgo, contribuyendo así a su éxito a mediano y largo plazo.

En el sector de los servicios son muy pocas las investigaciones relacionadas con este tema por lo cual se evidencia que hay un desconocimiento sobre él.

Los antecedentes referidos evidencian el escaso conocimiento acerca de las herramientas de la Gestión de la Tecnología y la Innovación en las entidades turísticas esencialmente, lo que provoca que no exista una adecuada coordinación y búsqueda de acciones innovadoras que contribuyan a una mejor gestión empresarial.

Teniendo en cuenta lo antes mencionado, el objetivo general, de la investigación consistió en:

Establecer el estado actual de su capacidad innovadora mediante la aplicación de una herramienta de Gestión de la Tecnología y la Innovación en la entidad hotelera objeto de estudio y proponer acciones que tributen a su mejora, para lograr una mejor Gestión Empresarial.

Este objetivo fue desglosado en los objetivos específicos siguientes:

- Plantear la base teórico-referencial relacionada con la Gestión de la Tecnología y la Innovación.

- Aplicar la herramienta que permita medir el grado de liderazgo de la entidad.

- Analizar los resultados de la herramienta aplicada y proponer acciones que tributen al mejoramiento del desempeño de la entidad y el logro de una mejor gestión empresarial.

Materiales y métodos.

Para medir el liderazgo se utilizó el instrumento apropiado, que es una novedad científica que radica en la puesta en práctica de un cuestionario para medir el Liderazgo hecho por los profesores del CETUM, ya validado, que responde al Proyecto de Investigación CITMA Territorial, que se desarrolla en el Polo Turístico de Varadero mediante diferentes investigaciones relacionadas con el:

- Diseño y aplicación del Cuadro de Mando Integral en entidades hoteleras.

- Diseño y Aplicación del Capital Intelectual. 
- Diseño y Aplicación del modelo de Gestión del Cliente Interno en entidades hoteleras.

Y las restantes son herramientas propuestas por el Dr. C. Rogelio Suárez Mella y las cuales se encuentran en su libro "El Reto".

Se aplica como instrumento fundamental el Software "GCV". Realizado a partir de la herramienta de igual nombre que son el fundamento sólido del libro "El Reto“, escrito por Rogelio Suárez Mella con el objetivo de facilitar y extender su uso por parte de los posibles usuarios.

Este Software contiene varios instrumentos con los que podemos conocer en que estadío se encuentran las empresas y de ahí trazarse un plan para su posterior análisis.

Descripción del instrumento que determina el Grado de Liderazgo.

El liderazgo hay que abordarlo como un proceso, del cual dependen las circunstancias y los propósitos, de ahí que cada persona puede ejercer el liderazgo en cierto momento y en otros no.

El liderazgo es visto como el proceso de gerencial exitosamente cualquier tipo de cambio organizacional: empresarial, político o social.

Tabla 1. Cuestionario de liderazgo propuesto por el DETUM.

Estimado compañero (a): el siguiente grupo de enunciados está relacionado con sus opiniones sobre liderazgo. Por favor, demuestre hasta qué punto Ud. cree que las personas que asumen ese papel poseen las características descritas. Utilice la escala presentada a continuación. Si usted está totalmente de acuerdo (TA) en que estas personas poseen una característica dada, sitúe un 7 en la línea que corresponde al enunciado. Si usted está totalmente en desacuerdo (TD), sitúe el 1 en la línea que corresponda al enunciado. Si sus opiniones de encuentran entre los extremos, sitúe un número entre 1 y 7 en la línea correspondiente, de modo que refleje realmente su opinión. No existen respuestas buenas o malas, ni verdaderas o falsas.

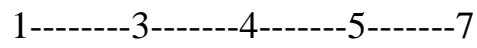

TD TA

\#

Ítems del cuestionario Liderazgo

Valor de la Escala

Toma iniciativas y cumple con sus responsabilidades, por lo que

1 constituye un ejemplo personal, tratando de buscar nuevas formas para que sus planteamientos sean aceptados e insiste hasta alcanzar lo que quiere. 
Posee valor moral, físico, mental y espiritual, demuestra fuerza en las

2

ideas, son las bases sobre las que sustenta su conducta, sus opiniones y sus ideas.

Posee entusiasmo, optimismo y compromisos para llevar a cabo las misiones, tiene carácter, posee algo especial, mezcla de carisma y personalidad.

4 Busca el mejor rendimiento de las personas.

5 Motiva a los demás a seguirle y que se sumen a las iniciativas, provocando la unidad y todos ponen manos a la obra.

6 Es capaz de identificar a los empleados más valiosos para la organización.

7 Permite que los mejores no se vayan o se desmotiven, ausencia total de odio hacia cualquier ser humano.

Brinda las herramientas e instrumentos para lograr el éxito y la diversidad de opiniones de forma que los planes los organiza de forma que las personas sean atraídas a lo que decida.

Expresa su visión en términos simples y en un ambiente de intercambio,

9 demuestra claridad, verdad, coherencia en una conversación necesita exponer sus ideas y transmitirlas.

10 Es accesible y crea las posibilidades para ello.

Crea un clima psicológico en el cual la información se percibe como un recurso y no como una amenaza. Desarrollando así sensibilidad para ser escuchado, Las personas atienden lo que dice y en ocasiones eleva el tono de voz.

Brinda a todas las personas el tiempo razonable y la oportunidad de

adaptarse y desarrollarse. Lo que permite la justicia de todos porque todo se hace y se decide conjuntamente.

Se mantiene al tanto de las mejores ideas de la gente que dirige porque el

13 deber es aprende, acepta las ideas de otros, siempre trata de comprender las ideas de los demás y ayuda a las personas a realizar las metas que emanan de la visión.

14 Enfrenta a personas aunque estas se enojan, desde la niñez estuvo al frente de grupos.

Dedica la mayor parte del tiempo a las personas, muestra desprendimiento. 
Es capaz de asumir riesgo, enfrenta retos y aporta soluciones a los

16

problemas, muestra rigor personal, cambia de opinión hace cuando está equivocado o los argumentos que le da son lógicos.

Siempre está dispuesto a romper con la rutina, demuestra gran capacidad

de ajustarse y adaptarse al cambio, es revolucionario, si tiene que hablar

en público expresa sus opiniones de forma clara.

Realiza y propicia que las personas hagan los cambios necesarios para

adaptarse al nuevo entorno. Trata de que los demás sigan sus instrucciones y se enfada si no logra los resultados.

Es capaz de prever y anticipares a las situaciones que producen el cambio.

Piensa que el orden es imprescindible: necesita mantener el orden y obliga a que los demás lo sean.

Prioriza el rendimiento y el cambio en la dirección de la organización.

Ejerce un liderazgo práctico, orientado hacia las personas. Nunca ha

21 dejado de sentirse un ser humano, aunque gusta que las personas cumplan con lo que manda y suele ser obedecido.

22 Comportamiento ético.

Fuente: Elaboración propia.

Para calcular el peso de cada ítem del cuestionario existen deferentes métodos como son:

- Métodos de Comparaciones Pareadas: El Triángulo de Füller y el Proceso Analítico Jerárquico, entre otros.

- Método del Coeficiente de Concordancia de Kendall.

- Método de Escalas de Proporción.

En este caso se utilizó El Triángulo de Füller, para la realización de este método se necesita un experto que se selecciona a través del método de selección de los expertos.

Primera fase del cuestionario:

En esta primera fase se obtiene información que permite calcular el coeficiente de conocimientos o de información que posee el Experto en relación con el problema que se quiere resolver. Los ítems que aparecen en la primera columna han sido obtenidos de dos fuentes: la literatura consultada acerca de las competencias que debe poseer un sujeto para calificarlo como experto en el ámbito de un problema concreto, y la opinión de personas con trabajo reconocido.

Es decir, la opinión que tienen personas expertas acerca de las características que debe poseer un experto en lo que ha conocimiento se refiere y otras. Los autores han incluido una segunda 
columna donde se consigna la prioridad o peso que posee la característica dada en un experto concreto. Esto suple la tradicional escala utilizada por otros autores donde sólo se obtiene un valor escala asignado por el propio evaluado.

La tercera columna expresa la votación que realiza el propio evaluado o la percepción que tiene un tercero acerca de la presencia o no de la característica en el sujeto objeto de evaluación. La información así obtenida permite calcular el mencionado coeficiente.

Segunda fase del cuestionario:

En esta fase se obtiene la información que posibilita calcular el coeficiente de argumentación. Esta información está estrechamente vinculada con el coeficiente que se calcula en la primera fase. Las fuentes de conocimientos se clasifican según criterios altos, medios y bajos asignando un valor determinado a cada fuente. La suma de esos resultados da el valor total del coeficiente.

Se obtiene el Coeficiente de competencia $\mathrm{K}$ del experto, al promediar la puntuación correspondiente a cada una de las partes del cuestionario, se propone que este coeficiente debe estar entre $0.8<\mathrm{K}<1$, con el objetivo de hacer una selección rigurosa de los profesionales dispuestos a participar de la investigación.

El procedimiento para la selección de expertos considera tres etapas fundamentales:

- Determinación de la cantidad de expertos.

- Confección de la lista de expertos.

- Obtener el consentimiento del experto en su participación.

Para encontrar personas «expertas» podrían ser aquellas que desempeñan el trabajo, formadores, especialistas en seguridad, mandos, dirección y personas del equipo de proyectos y diseño. Para que una persona pueda ser etiquetada como «experta» debe poseer un conocimiento profundo de la tarea o actividad que será objeto de análisis y valoración y tiene que estar familiarizada con el sistema en el que esta se desarrolla. Asimismo, los expertos a los que se recurre tienen que ser capaces de traducir su valoración en términos de probabilidades.

Se debe tener previsto el modo en que se recogerán los juicios de los expertos y tener preparados, si es el caso, cuestionarios, escalas de evaluación, etc. La precisión en estos es esencial para que los resultados no queden sesgados por fallos de comprensión. También se deben preparar las instrucciones que se van a dar a los jueces o expertos, de modo que comprendan con claridad cuál es el objetivo de su ejercicio de evaluación.

Después que se seleccione el experto, se pasa a hallar el valor del peso de cada ítem por el Triángulo de Füller que fue la herramienta elegida. 
Para el análisis de los datos del cuestionario se utilizó el arreglo matricial para obtener Valor Percibido Total (VpT) normal y estos resultados de la matriz se colocan en el SPSS para hallar la correlación entre las votaciones a través del Perfil Factorial de Percepción, que es un modo gráfico de líneas a través del cual se representa las correlaciones de atributos /dimensiones con el grado de presencia percibido de los mismos en una empresa o conjunto de ellas. Se puede construir fácilmente en Microsoft Excel utilizando la opción gráfica correspondiente.

Para el cálculo del valor percibido se utilizó el Método para hallar la muestra con sus respectivos cálculos (tamaño de la muestra, varianza poblacional y muestreo aleatorio).

\section{Resultados.}

Se utilizó el cuestionario de liderazgo hecho por el CETUM y se pasó a hallar el peso de los ítems del cuestionario a través de la herramienta Triangulo de Füller, como experto se seleccionó al Jefe de Recursos Humanos después de haber pasado por el método de selección de los expertos.

Después de aplicar la encuesta de liderazgo a la muestra seleccionada y hallar el valor percibido, se colocaron los valores de la matriz en el sistema estadístico SPSS para verificar la existencia de correlación entre las votaciones, lo que arrojo que si existe debido a que el valor de alpha es mayor que 0.4 y luego se llevaron los resultados de la matriz al grafico de perfil factorial de percepción.

El comportamiento de esta variable se expresa a través de un valor medio geométrico de 4.2 lo que es ligeramente superior al valor neutral de la escala. En ello incide tal y como se muestra la distribución de frecuencia de las evaluaciones. Se observa lo siguiente:

- 5 atributos están en la franja de 4,4 a 4,5 (el no-6 es capaz de identificar a los empleados más valiosos), el no-7 (permite que los mejores no se vayan o se desmotiven, ausencia total de odio hacia cualquier ser humano), el no-14 (enfrenta a personas aunque estas se enojan, desde la niñez estuvo al frente de grupos), no16 (es capaz de asumir riesgo, enfrenta retos y aporta soluciones a los problemas, muestra rigor personal, cambia de opinión hace cuando está equivocado o los argumentos que le da son lógicos), no-17 (siempre está dispuesto a romper con la rutina, demuestra gran capacidad de ajustarse y adaptarse al cambio, es revolucionario, si tiene que hablar en público expresa sus opiniones de forma clara).

- En la zona de 4,3 a 4,4 se encuentran 11 atributos para un total del 50\%, se encuentra el no-1 (toma iniciativas y cumple con sus responsabilidades, por lo que constituye un ejemplo personal, tratando de buscar nuevas formas para que sus planteamientos sean aceptados e insiste hasta alcanzar lo que quiere), el no -2 (posee valor moral, físico, mental y espiritual, demuestra fuerza en las ideas, son 
las bases sobre las que sustenta su conducta, sus opiniones y sus ideas), el no-3 (posee entusiasmo, optimismo y compromisos para llevar a cabo las misiones, tiene carácter, posee algo especial, mezcla de carisma y personalidad), el no-8 (brinda las herramientas e instrumentos para lograr el éxito y la diversidad de opiniones de forma que los planes los organiza de forma que las personas sean atraídas a lo que decida), el no-9 (expresa su visión en términos simples y en un ambiente de intercambio, demuestra claridad, verdad, coherencia en una conversación necesita exponer sus ideas y transmitirlas), el no-12 (brinda a todas las personas el tiempo razonable y la oportunidad de adaptarse y desarrollarse. Lo que permite la justicia de todos porque todo se hace y se decide conjuntamente), el no-13 (se mantiene al tanto de las mejores ideas de la gente que dirige porque el deber es aprende, acepta las ideas de otros, siempre trata de comprender las ideas de los demás y ayuda a las personas a realizar las metas que emanan de la visión), el no-15 (dedica la mayor parte del tiempo a las personas, muestra desprendimiento), el no-18( realiza y propicia que las personas hagan los cambios necesarios para adaptarse al nuevo entorno. Trata de que los demás sigan sus instrucciones y se enfada si no logra los resultados), el no-19 (es capaz de prever $\mathrm{y}$ anticipares a las situaciones que producen el cambio. Piensa que el orden es imprescindible: necesita mantener el orden y obliga a que los demás lo sean), el no-22 (comportamiento ético),

- En el rango de 4,2 a 4,3 se encuentran 5 atributos, el no-4 (busca el mejor rendimiento de las personas), el no-10 (es accesible y crea las posibilidades para ello), el no-11(crea un clima psicológico en el cual la información se percibe como un recurso y no como una amenaza. Desarrollando así sensibilidad para ser escuchado, Las personas atienden lo que dice y en ocasiones eleva el tono de voz), el no-20 (prioriza el rendimiento y el cambio en la dirección de la organización), el no- 21(ejerce un liderazgo práctico, orientado hacia las personas. Nunca ha dejado de sentirse un ser humano, aunque gusta que las personas cumplan con lo que manda y suele ser obedecido). En la zona de 4 a 4,1 el no-5 (motiva a los demás a seguirle y que se sumen a las iniciativas, provocando la unidad y todos ponen manos a la obra).

En general, el valor percibido de liderazgo en el hotel es bajo, con un valor de 4,33 porque no se busca el mejor rendimiento de las personas, no motiva a los demás a seguirle, no es accesible, crea un clima psicológico en el cual la información se recibe como una amenaza, no se prioriza el rendimiento ni el cambio en la organización, no ejerce un liderazgo práctico orientado a las personas.

Acciones propuestas:

- Motiva a las personas a unírsele en el cumplimiento de metas trazadas buscando lo mejor de estas. 
- Ser más abierto a los trabajadores y escucharlos para darle solución a sus problemas.

- Crear un clima en el cual la información sea recibida como un recurso para mejorar su rendimiento en su puesto de trabajo y que no se perciba como una amenaza.

- Ejercer un liderazgo orientado a las personas y priorizar el rendimiento.

\section{Conclusiones.}

- El uso de este instrumento es válido para cualquier entidad. El liderazgo es un proceso del que dependen las circunstancias y los propósitos de toda organización, ya sea empresarial, político o social, por lo que su medición es parte importante para el éxito a mediano y largo plazo.

- El valor de liderazgo percibido es bajo, los trabajadores no son motivados a seguir al líder por lo que no tienen un mejor rendimiento, el clima es tenso y no se ejerce un buen liderazgo, por lo que se hace necesario la toma de acciones propuestas para crear un mejor clima laboral, las personas se sientan motivadas y con un liderazgo orientado a las mismas.

\section{Referencias bibliográficas.}

Actualización de los lineamientos de la Política Económica y Social del Partido y la Revolución para el periodo 2016 - 2021 aprobados en el 7mo Congreso del Partido en abril de 2016 y por la Asamblea Nacional del Poder Popular en junio de 2016.

Albornoz, M. (2009). Indicadores de innovación: las dificultades de un concepto en evolución. Revista CTS, No. 13. vol. 5: 9-25.

Alonso Gámez, L. (2015). La Vigilancia Tecnológica como instrumento para la construcción de un observatorio. Ingeniería Industrial Tesis en opción al título de Ingeniería Industrial, Universidad de Matanzas "Camilo Cienfuegos". Cuba.

Arias, M \& Zenea, M. (marzo de 2018). Calidad directiva: Liderazgo y equipos de dirección. En F. Borrás (Presidencia), Desarrollo de empresas: una agenda para su gestión. Ponencia llevada a cabo en 3er Congreso Internacional Gestión Económica y Desarrollo.

Blanco, H. (marzo de 2018). Soporte para la Innovación en la empresa. En F. Borrás (Presidencia), Desarrollo de empresas: una agenda para su gestión. Ponencia llevada a cabo en 3er Congreso Internacional Gestión Económica y Desarrollo.

Blanco, H. (marzo de 2018). La gestión de la Innovación en las empresas estatales cubanas. ¿Asignatura pendiente? En F. Borrás (Presidencia), Desarrollo de empresas: una agenda para su gestión. Ponencia llevada a cabo en 3er Congreso Internacional Gestión Económica y Desarrollo.

Bermúdez Estrada, A. and N. R. Lara Coba (2011). Propuesta de diseño de un Sistema de innovación empresarial para una empresa del sector textil-confección. Administración de empresa. Tesis de grado, Pontificia Universidad Javeriana. 
CITMA (2013). Política para la reorganización del Sistema de Ciencia, Tecnología e Innovación.

CITMA (2015). Bases para el otorgamiento de la condición de Entidad Innovadora de la provincia de Matanzas. Matanzas: 11.

Comunidad de Madrid (s. a). El sistema regional de I+D+I de la Comunidad de Madrid. D. G. d. Investigación, C. d. Educación and C. d. Madrid. Instituto de Análisis Industrial y Financiero; Universidad Complutense de Madrid.

Decreto No. 281 (2007). Decreto No. 281. 1682-7511. G. O. d. 1. R. d. Cuba. La Habana, Cuba, Ministerio de Justicia. 501: 325, 327.

EAE Business School. (2018). Las 5 herramientas imprescindibles de management que todo directive debe dominar. Recuperado de http://www.eae.es

European Communities. (2005). Oslo Manual: Guidelines for Collecting and Interpreting Innovation Data, Third Edition. Recuperado de http://www.tragsa.es

Gonzales, Lázaro. (2015). Procedimiento para la creación del sistema de Gestión de la Innovación de la UEB Aeropuerto Juan Gualberto Gómez. Tesis de Diploma. Universidad de Matanzas. Cuba.

Jiménez, Bisleivys. (2011). Procedimiento de evaluación y mejora de la Gestión de la Tecnología y la Innovación en hoteles todo incluido. Tesis Doctoral. Universidad de Matanzas. Cuba.

Martin González, A. (2015). Implantación de un procedimiento de gestión y mejora de procesos en la UEB Aeropuerto Juan Gualberto Gómez. Ingeniería Industrial, Universidad de Matanzas.

Santana, E. (marzo de 2018). La capacitación y la calidad directiva. Un enfoque prospectivo. En F. Borrás (Presidencia), Desarrollo de empresas: una agenda para su gestión. Ponencia llevada a cabo en 3er Congreso Internacional Gestión Económica y Desarrollo.

Sitio Web: Roberto A Frías Jiménez, Mahe González Arias, Olivia M Oliver Gil. Diseño y validación de un Modelo de Gestión del Cliente Interno en Procesos Hoteleros. Universidad de Matanzas.

Suárez Mella, R. et al. (2001). El Reto. Gestión de vitalidad en entornos competitivos. Editorial Academia, Ciudad de La Habana.

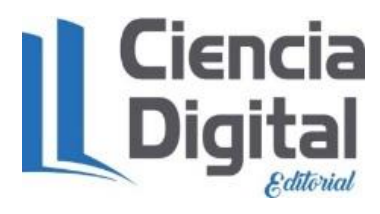




\section{Para citar el artículo indexado}

Mesa Ramos, D., Jiménez Valero, B., \& Bordón Gómez, Y. (2020). Aplicación de un instrumento para medir el grado de liderazgo en el hotel Villa Cuba. AlfaPublicaciones, 1(1). https://doi.org/10.33262/ap.v1i1.18

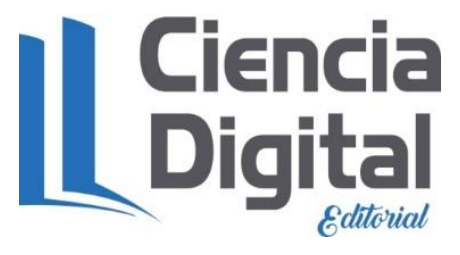

El artículo que se publica es de exclusiva responsabilidad de los autores y no necesariamente reflejan el pensamiento de la Revista Alpha Publicaciones.

El artículo queda en propiedad de la revista y, por tanto, su publicación parcial y/o total en otro medio tiene que ser autorizado por el director de la Revista Alpha Publicaciones.
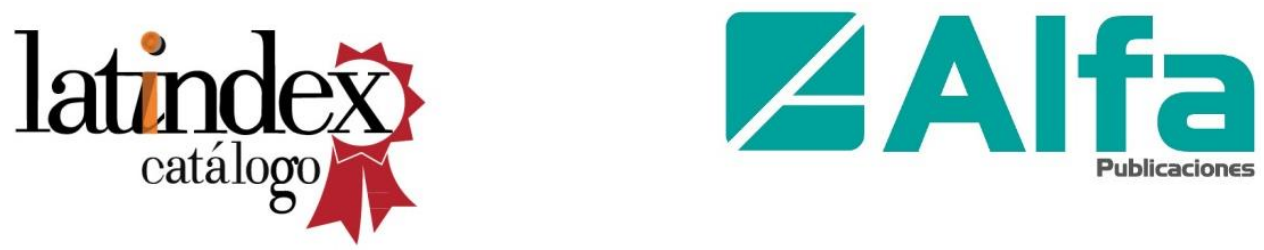